\title{
Aggressive Angiomyxoma: An Unusual Female Pelvic Tumour. Report of Three Cases and Review of the Literature
}

\author{
Juan Antonio Martín-Cartes, Manuel Bustos-Jiménez, María Jesús Tamayo-López, María del \\ Carmen Palacios-González, Virginia Gómez-Cabeza de Vaca, Antonio Muñoz Ortega \\ Division of Surgery, Abdominal Wall Surgery Unit, Seville, Spain \\ E-mail:jumarcar@telefonica.net \\ Received July 7, 2010; revised August 1, 2010; accepted August 10, 2010
}

\begin{abstract}
Aggressive angiomyxoma was first described in 1983 by Steeper and Rosai, and fewer than 150 cases have been reported in the world medical literature. It is a soft-tissue tumour of the pelvis and perineum. The recurrence rate is high, and often extensive resections are performed with considerable morbidity. These tumours are benign, locally infiltrative mesenchymal neoplasms with a predilection for the female pelvis and perineum and they usually tend to recur. Furthermore, these tumours often reach too large dimensions before becoming clinically symptomatic; their incidence is higher in women of the reproductive age group; however a few cases of its occurrence outside the pelvis have also been reported. In this study, we reported three cases with aggressive pelvic angiomyxoma treated with surgical methods and used an approach that described by Kraske in order to get access to lower rectal cancers. Accurate preoperative diagnosis should alert the surgeon to the need for wide excision, which is essential for prevention of local recurrence.
\end{abstract}

Keywords: Aggressive Angiomyxoma, Soft Tissue Tumour, Perineum and Pelvis Tumour

\section{Introduction}

Aggressive angiomyxoma is a rare tumour of mesenchymal origin first described in 1983 by Steeper and Rosai [1-2] like a distinct mesenchymal tumour of the female pelvis and perineum. However, it has later been reported in males. In men, the tumour involves analogous sites including the scrotum and inguinal area and usually appears at an older age [3]. It's suspected there might be a relation with hormonal status that might explain a female to male ratio of slightly more than $6: 1$. Have been reported about 150 cases in the world medical literature [4-8].

These lesions are characterized as soft, non-encapsulated tumours with finger-like projections infiltrating the surrounding soft tissues. The tumour presents as a large multilobular or polypoid mass or swelling. On gross examination it is rubbery and white or soft and gelatinous. The tumour grows slowly, and its benign nature is suggested by the histology and by the fact that it shows no tendency to metastasize [9]. Unlike malignant neoplasms, do not metastasize, and, histologically, they demonstrate bland nuclei without atypia or mitotic activity. However it usually tends locally to recur [10]. Correlation between tumour size and subsequent risk of recurrence has not been demonstrated [11].

Surgery is usually the first line of treatment, radical surgery with wide margins and long-term follow-up is advised. There has been some interest in the potential use of hormonal manipulation to manage those tumours after surgery. Intuitively, hormonal suppression seems to be a plausible treatment option because these tumours occur predominantly in premenopausal women of reproductive age, may grow rapidly during pregnancy, and have been shown to express immunohistochemical positivity for estrogenic and progesteronic receptors [12].

\section{Material and Methods}

From January'09 to April'10, three women with aggressive angiomyxoma were managed in our Unit. Two of these cases were directly managed in our centre while the other had previously been operated in Rumania.

We also performed a Medline search from 2006 to 
2010 with the key words "aggressive angiomyxoma" and reviewed literature we had found.

This Medline search reported up to 150 cases, 112 cases of this rare neoplasm were reported in women if limited to the perineum and pelvis.

The most relevant details for the three patients managed in our Unit are detailed below.

Patient 1. A 50-year-old Rumanian female. In 2006, in Rumania, she was operated on her pelvic area using a trans-abdominal approach and a great pelvic mass was resected. The mass was diagnosed as an aggressive angyomixoma postoperatively.

In 2009, after she arrived in Spain, she presented again symptoms such as presented with a long history of a vague, poorly defined, painless swelling over the right ischial tuberosity. On examination, in cases 1 and 3 , the swelling was ill defined, non-tender, and difficult to palpate. MRI scans showed large and abnormal soft tissue masses $(9.6 \times 4.5 \times 4.5 \mathrm{~cm})$ within the pelvis. Those masses were iso-intense, but sometimes became very hyperintense. At present, after 12 months no recurrence.

Patient 2. A 36-year-old female. She presented a 3-year history of swelling left sided in vulva and perineum, big and evident mass on examination and palpation. She had changed her bowel habit. MRI scans showed a big mass $(24 \times 11 \times 12.3 \mathrm{~cm})$ in minor pelvis extending into right ischiorectal fossa, left paravaginal area and perineum (Figures 1 and 2). After 9 months no recurrence observed.

Patient 3. A 28-year-old female. She presented a 2-year history of swelling left sided in vulva and perineum, big mass on examination and palpation. She had changed her bowel habit, suffered from urinary urgency and had been treated with hormonal stimulators in order to donate her oocytes. MRI scans showed a big mass (20 $\times 10 \times 9 \mathrm{~cm}$ ) affecting her minor pelvis and extending into right ischiorectal fossa and left paravaginal area. After 9 months no recurrence observed.

In all cases the diagnosis of aggressive angiomyxoma was made only after initial surgery.

Preoperative misdiagnosis of aggressive angiomyxoma is not infrequent because this rare neoplasm has no universally typical symptoms.

All cases presented with a long history of a vague, poorly defined, painless swelling over the right ischial tuberosity. On examination, in cases 1 and 3, the swelling was ill defined, non-tender, and difficult to palpate, in case 2 the mass was evident enough. Clinically the initial differentials were of lipoma, liposarcoma or pelvic floor hernia.

MRI scans showed large and abnormal soft tissue masses within the pelvis. Those masses were iso-intense, but sometimes became very hyperintense, with much whorled solid tissue within. There was no fluid or fat within them. The masses were extended from the pubic symphysis until to the rectum and extended as far as S1 level superiorly, retroperitoneally and inferiorly as far as the level of the anal sphincter. The MRI appearances were suggestive of an unusual mesenchymal tumour.

Abdominal and pelvic CT scan, and transvaginal ultrasound and intravenous urography (IVU) both confirmed the well-circumscribed appearance of the mass and deviation of rectum, uterus, ureters and bladder.

Surgery revealed a large mass located in the lower abdomen, the left pelvis extending to the left ischio-rectal region. The tumour had closed contact with muscles. Complete excision was performed by a Kraske approach. Coxigeal and partial sacral excision was only made in case 3.

Patients 1 and 2 stayed at hospital for a week.

Cut section in all cases revealed a gelatinous soft and greyish coloured tumour with a fine capsule and no lobes, necrosis or cystic degeneration. Excised tumours had a gelatinous composition and weighed 500, 1030 and $495 \mathrm{gr}$ (Figures 1-3).

Microscopic examination showed a relatively hypocellular tumour with myxoid stroma containing numerous vessels, some with thick walls. The cells showed regular nuclei and stellate fine cytoplasm. There was no cytologic atypia, no atypical mitotic features or discernible mitotic activity and no evidence of coagulative tumor cell necrosis either.

Immunohistochemical studies showed the cells to be positive for vimentine and dismin. There was a strong positivity for estrogenic and progesteronic receptors. In our cases show the cells to be positive for vimentine and focally positive for dismin and smooth muscle actin.

That is why all cases the masses were diagnosed as aggressive angiomyxoma. The recurrent tumours showed similar histological characteristics to the primary ones.

MRI scans showed large and abnormal soft tissue masses within the pelvis. Those masses were iso-intense, but sometimes became very hyperintense, with much whorled solid tissue within. There was no fluid or fat within them. The masses were extended from the pubic symphysis until to the rectum and extended as far as S1 level superiorly, retroperitoneally and inferiorly as far as the level of the anal sphincter. The MRI appearances were suggestive of an unusual mesenchymal tumour.

Abdominal and pelvic CT scan, and transvaginal ultrasound and intravenous urography (IVU) both confirmed the well-circumscribed appearance of the mass and deviation of rectum, uterus, ureters and bladder.

\section{Results}

The patients 1 and 2 made an uneventful recovery, and went home 7 days later. The other suffered from a partial 
skin necrosis and she stayed at hospital for a second postoperative week. At present, 5-12 months' follow-up, there was no evidence of recurrence either clinically or on imaging studies.

\section{Discussion}

Aggressive angiomyxoma is a rare benign tumour that arises from the connective tissue of the perineum or

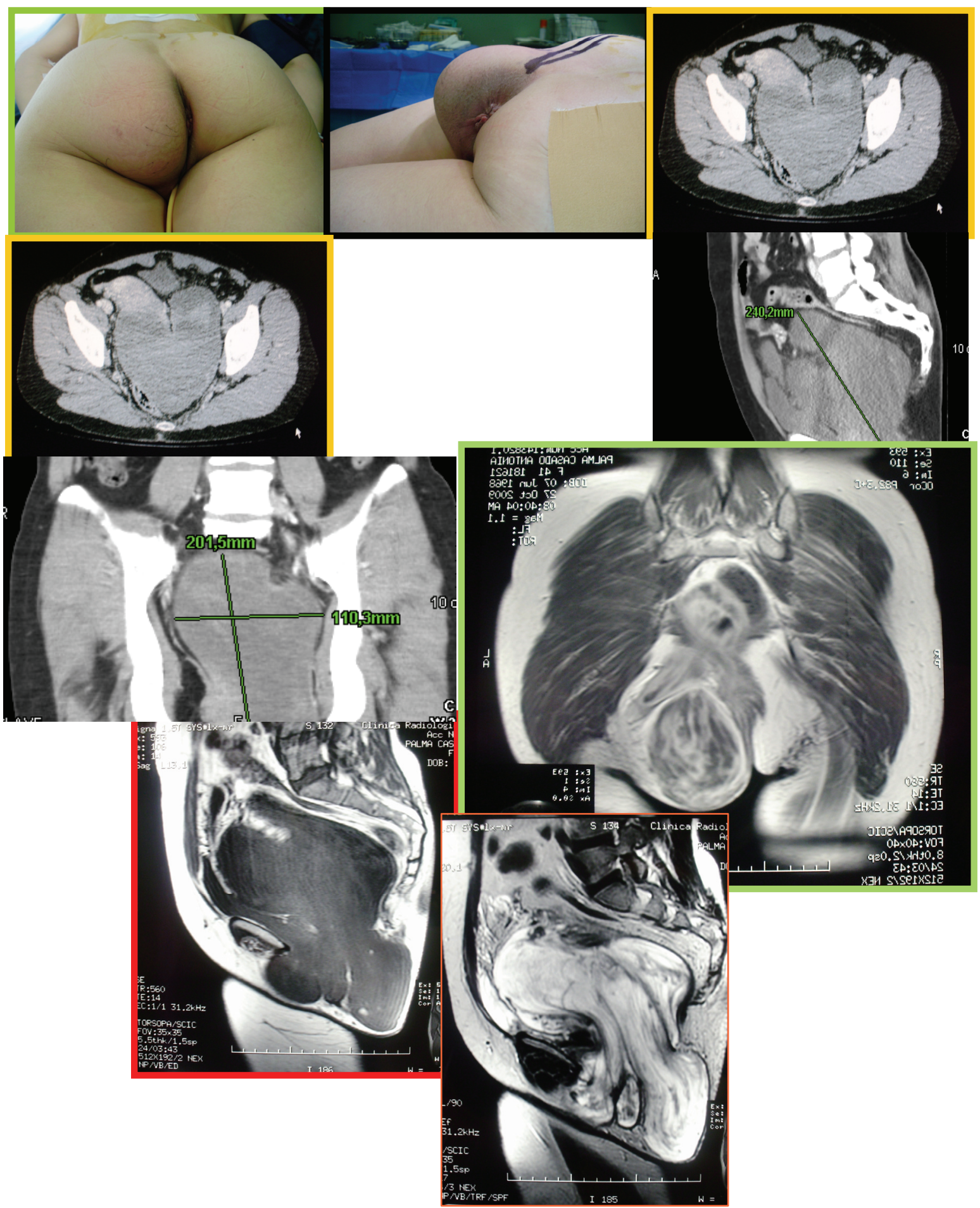

Figure 1. Case 1. Pelvic mass on physical examination and its images. 

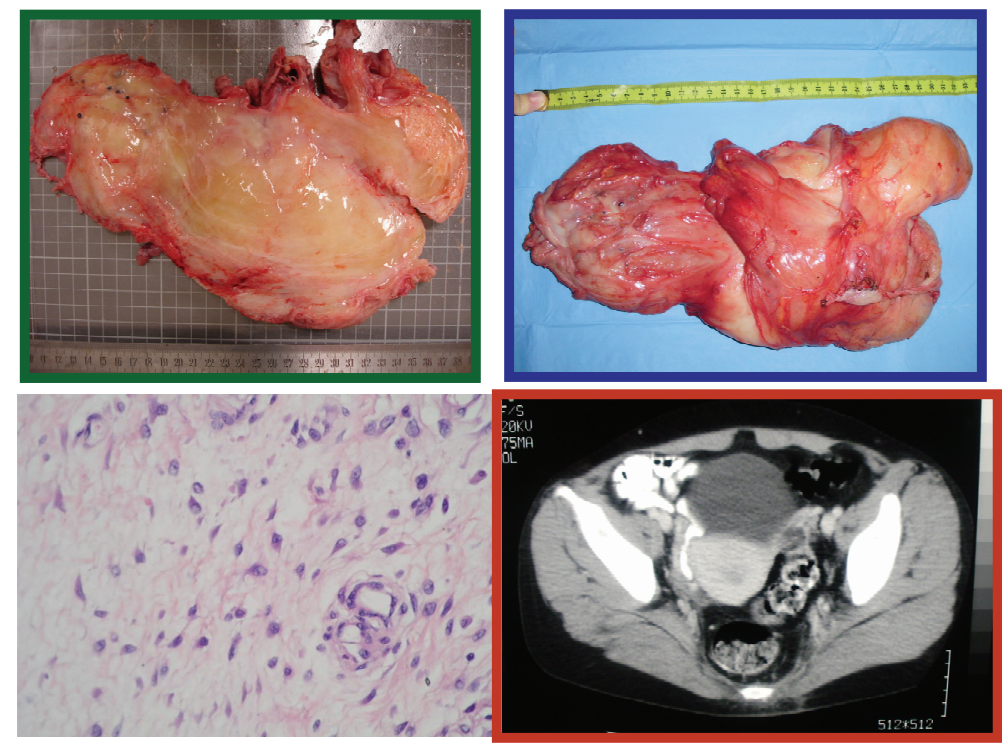

Figure 2. Case 2. The excised mass and postoperative tomography.
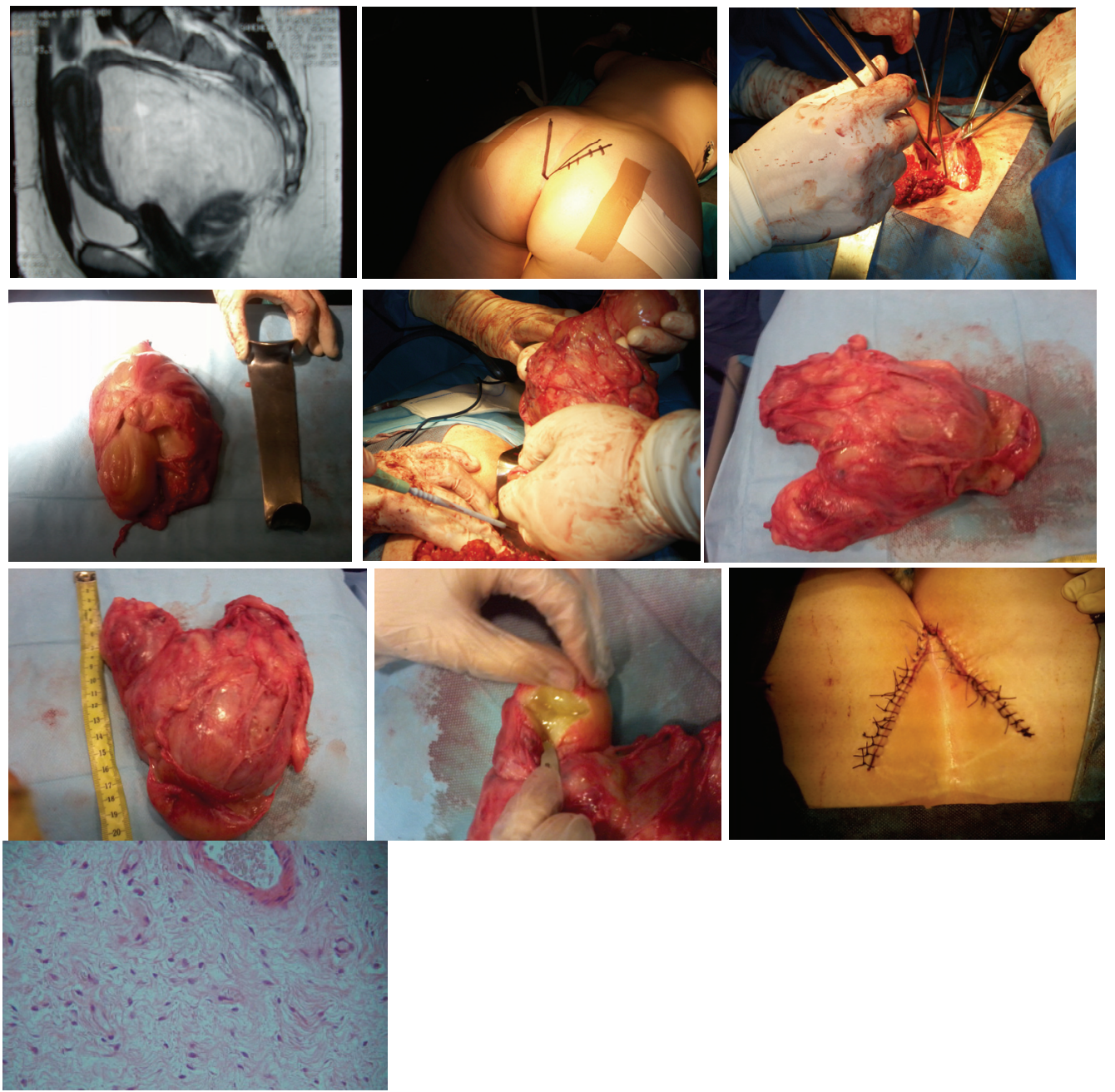

Figure 3. Case 3. Preoperative CT, the excised specimen and the final appearance. 
lower pelvis. The rarity of this condition makes the preoperative diagnosis fairly difficult. It has been related to hormonal activity; our cases 1 and 2 were pre- menopausal females, and the other had undergone medical hormonal stimulation treatment in order to donate her ovules for five years.

Complete surgical excision with tumour-free margins is the main objective. This surgery is challenging because of the infiltration and the difficult dissection. In the past, most authors advocated wide excision even if genito-urinary and digestive tract resections were necessary.

On the one hand, local excision of villous adenomas and other benign rectal lesions has been accepted for decades, and the Kraske approach for management of these lesions has been recommended by several authors. Surgical resection of rectal disease via a posterior approach was first described by Kraske at the Fourteenth Congress of the German Association of Surgeons in 1885. In his initial description, Kraske advocated removingtumors of the middle rectum by excising the coccyx and a portion of the sacrum. The original procedure described division of the left gluteal muscle insertions, the left sacrotuberous and sacrospinous ligaments, and the left fourth and fifth sacral nerves.

Furthermore, that approach may be useful to excise pelvic masses like the above described in order to reach both upper and lower ends.

On the other hand, the posterior approach to the rectum utilizing the Kraske procedure provides a better exposure for distal rectum (5-10 $\mathrm{cm}$ from the anal verge) so that we can locate it on surgical procedure [13-16].

Although Mera-Velasco [17] has reported the utility of laparoscopic approach, we do not agree with him. We do not consider doing that approach so as not to have to fragment the excised specimen. Moreover, it is necessary to have in mind that releasing the lower end of those masses is usually the most difficult step in the surgical procedure.

Because of abdominal and pelvic organ manipulation, hospital stays are generally longer, and patients are at higher risk of developing problems such as deep venous thrombosis and pulmonary embolism while they await the return of bowel function after procedure.

To sum up, aggressive angiomyxoma should be distinguished from other, more common neoplasms of the pelvic tissues. When the mass is not well defined or has atypical behaviour, improving preparation before surgery, and direct the surgeon toward complete excision might be the best option.

\section{References}

[1] I. J. M. Han-Geurts, A. N. van Geel, L. van Doorn, et al.,
"Aggressive Angiomyxoma: Multimodality Treatments can Avoid Mutilating Surgery," European Journal of Surgical Oncology, Vol. 32, No. 10, 2006, pp. 1217-1221.

[2] T. A. Steeper and J. Rosai, “Aggressive Angiomyxoma of the Female Pelvis and Perineum: Report of Nine Cases of a Distinctive Type of Gynaecologic Soft-Tissue Neoplasm," American Journal of Surgical Pathology, Vol. 7, No. 5, 1983, pp. 463-475.

[3] K. A. Behranwala and J. M. Thomas, "Aggressive' Angiomyxoma: A Distinct Clinical Entity," European Journal of Surgical Oncology, Vol. 29, No. 7, 2003, pp. 559563.

[4] A. Mathieson, S. Chandrakanth, G. Yousef, et al., "Aggressive Angiomyxoma of the Pelvis: A Case Report," Canadian Journal of Surgery, Vol. 50, No. 2, 2007, pp. 228-229.

[5] P. M. Magtibay, Z. Salmon, G. L. Keeney, et al., "Aggressive Angiomyxoma of the Female Pelvis and Perineum: A Case Series," International Journal of Gynecological Cancer, Vol. 16, No. 1, 2006, pp. 396-401.

[6] K. Haldar, I. E. Martinek and S. Kehoe, "Aggressive Angiomyxoma: A Case Series and Literature Review," European Journal of Surgical Oncology, Vol. 36, No. 4, 2010, pp. 335-339.

[7] S. Dove, P. Remoue, E. Fondrinier, et al., "Unusual Female Pelvic Tumour: Aggressive Angiomyxoma," European Journal of Obstetrics \& Gynecology and Reproductive Biology, Vol. 137, No. 3, 2008, pp. 123-125.

[8] E. L. Nava Flores, M. A. Alvarez Blanco, J. Figueroa Vadillo, et al., "Aggressive Angiomyxoma of the Vulva. Case Report and Literature Review," Ginecologia Y Obstetricia de Mexico, Vol. 77, No. 7, 2009, pp. 487-490.

[9] A. Wiser, J. Korach, W. H. Gotlieb, E. Fridman, et al., "Importance of Accurate Preoperative Diagnosis in the Management of Aggressive Angiomyxoma: Report of Three Cases and Review of the Literature," Abdominal Imaging, Vol. 31, No. 3, 2006, pp. 383-386.

[10] H. Adwan, P. D. Kamel and G. Glazer, "A Solitary Encapsulated Pelvic Aggressive Angiomyxoma," Annals of The Royal College of Surgeons of England, Vol. 86, No. 6, November 2004, pp. W1-W3.

[11] M. Suleiman, C. Duc, S. Ritz and S. Bieri, "Excision of large Aggressive Angiomyxoma in a Woman: Irradiation for Recurrent Disease," International Journal of Gynecological Cancer, Vol. 16, No. (Suppl. 1), 2006, pp. 356360.

[12] M. Onaitis, K. Ludwig, A. Perez-Tamayo, et al., "The Kraske Procedure: A Critical Analysis of a Surgical Approach for Mid-Rectal Lesions," Journal of Surgical Oncology, Vol. 94, No. 3, 2006, pp. 194-202.

[13] P. Gervaz, O. Huber, P. Buche, et al., "Trans-Sacral (Kraske) Approach for Gastrointestinal Stromal Tumour of the Lower Rectum: Old Procedure for a New Disease," Colorectal Disease, Vol. 10, No. 9, 2008, pp. 951-952.

[14] S. E. Wilson and H. E. Gordon, "Excision of Rectal Lesions by the Kraske Approach," American Journal of Surgery, Vol. 118, No. 2, 1969, pp. 213-217. 
[15] J. P. Arnaud, M. R. Eloy, G. Clendinnen, et al., "The Posterior Approach for Villous Tumors of the Rectum," American Journal of Surgery, Vol. 136, No. 2, 1978, pp. 273-275.

[16] J. Christiansen, "Excision of Mid-Rectal Lesions by the
Kraske Sacral Approach," British Journal of Surgery, Vol. 67, No. 9, 1980, pp. 651-652.

[17] S. Mera-Velasco, A. J. Cabello-Burgos, M. Ruiz-López, et al., "Laparoscopic Resection of an Aggressive Pelvic Angiomyxoma," Cirugia Espanola (in Spanish), 2009. 